\title{
Diversidad y distribución de mariposas Satyrinae (Lepidoptera: Nymphalidae) en la cuenca del río Coello, Colombia
}

\author{
Jack F. García-Perez, Leonardo A. Ospina-López, Francisco A. Villa-Navarro \& \\ Gladys Reinoso-Flórez \\ Grupo de Investigación en Zoología. Facultad de Ciencias. Universidad del Tolima (Colombia); Fax: (098) 2669182; \\ jackdroun@colombia.com,ospilope@hotmail.com, favilla@ut.edu.co,glareinoso1@hotmail.com
}

\author{
Recibido 30-V-2004. Corregido 02-V-2006. Aceptado 15-I-2007.
}

\begin{abstract}
Diversity and distribution of Satyrinae butterflies (Lepidoptera: Nymphalidae) in the Coello river basin, Colombia. We describe the patterns of diversity and distribution of the subfamily Satyrinae in the Coello river basin $\left(4^{\circ} 17^{\prime} 08^{\prime \prime} \mathrm{N}-74^{\circ} 35^{\prime} 36^{\prime \prime} \mathrm{W} ; 1899.31 \mathrm{~km}^{2}\right)$ from 433 to $3600 \mathrm{~m}$. Eleven sampling stations were located, in several ecosystems. The samples were collected during a period of 11 days, in March, May, July and October 2003, in three types of habitat (forest, forest edge and meadow). We collected at random, between 09:00 and 15:00 hr, using entomological nets. A total of 239 individuals (13 genera, 34 species) were collected. The most abundant species were in the genus Pedaliodes $(41.4 \%)$. Richness and diversity had high values in mountainous zones and paramo $(>2000 \mathrm{~m})$. The greater diversity was measured in the forest edge. The similarity analysis produced two groups: species from warm (Euptychia hesione and Hermeuptychia hermes) and middle (Pseudohaetera hypaesia and Taygetis celia) climate zones; and species from mountain and paramo (Lymanopoda obsoleta, Pedaliodes polusca and Eretris calisto). Rev. Biol. Trop. 55 (2): 645-653. Epub 2007 June, 29.
\end{abstract}

Key-words: Coello, Satyrinae, Pedaliodes, mountainous zones, paramo, similarity.

La familia Nymphalidae (Lepidoptera: Papilionoidea) contiene unas 7250 especies, siendo la familia más diversa de mariposas diurnas, y aproximadamente $42 \%$ de ellas son neotropicales (García-Robledo et al. 2002). De acuerdo con Amat et al. (1999) para Colombia se estiman 1182 especies de Nymphalidae, de las cuales 299 especies pertenecen a la subfamilia Nymphalinae, 295 especies a Ithomiinae y 225 a Satyrinae, siendo por lo tanto éstas tres subfamilias las más representativas. Para la cordillera Central la subfamilia Satyrinae es la primera en número de especies, con alrededor de 95 especies (Andrade y Amat 1996). Este grupo se caracteriza por las plantas hospederas que son exclusivamente monocotiledóneas, principalmente en Poaceae, Marantaceae, Arecaceae y Cyperaceae (García-Robledo et al. 2002), además en algunos briófitos (De Vries 1987).

En Colombia son pocos los estudios que se han realizado específicamente para la subfamilia Satyrinae, destacándose el de Andrade y Amat (1996), donde se da a conocer un primer listado de mariposas altoandinas de la cordillera Oriental de Colombia. Los Papilionoidea han sido estudiados por Álvarez (1993), quien realizó un inventario de mariposas diurnas en dos zonas de Risaralda, después Fagua (1999) estableció la variación de la riqueza y diversidad de mariposas y hormigas en un gradiente de la cordillera Oriental, Tobar (2000) estudió la composición regional y local de mariposas diurnas en diferentes relictos de bosque y en áreas abiertas en la parte alta de la cuenca del río Roble (Quindío), y García-Robledo et al. 
(2002) realizó un inventario midiendo la distribución y aspectos ecológicos de mariposas comunes de la cordillera Central.

Los indicadores biológicos, son definidos como las especies o grupos taxonómicos que pueden reflejar el estado de la biota en cuanto a biodiversidad, su relación con otras áreas geográficas, variación a lo largo de gradientes, endemismos o el grado de intervención humana (Fagua 2001).

Las mariposas en general son muy sensibles a los cambios de temperatura, humedad y radiación solar que se producen por disturbios en su hábitat, por lo cual el inventario de sus comunidades, a través de medidas de diversidad y riqueza, representa una herramienta válida para evaluar el estado de conservación o alteración del medio natural (Kremen et al. 1993, Fagua 1999). Este estudio describe la diversidad y composición anual de la subfamilia Satyrinae en la cuenca del río Coello, además de su posible uso como indicador biológico de zonas montanas y de Páramo.

\section{MATERIALES Y MÉTODOS}

Este estudio se realizó en la cuenca del río Coello, la cual se encuentra localizada en el centro del departamento del Tolima, flanco derecho de la cordillera Central a $4^{\circ} 17^{\prime}$ $08^{\prime} " \mathrm{~N}$ y $74^{\circ} 35^{\prime} 36^{\prime} \mathrm{W}$, abarcando un área de $1899.31 \mathrm{~km}^{2}$. La cuenca exhibe una gran variabilidad fisiográfica, presentando variados pisos térmicos, los cuales comprenden zonas cálidas: 280 a 1000 m.s.n.m., temperatura $24-28{ }^{\circ} \mathrm{C}$, bosque muy seco (bms-T) y seco tropical (bs-T), zonas templadas: 1000 a 2000 m.s.n.m., temperatura $18-24{ }^{\circ} \mathrm{C}$, bosque húmedo subtropical (bh-ST), zonas frías: 2000 a 3 000 m.s.n.m., temperatura $10-18{ }^{\circ} \mathrm{C}$, bosque húmedo montano bajo (bh-MB) y bosque muy húmedo montano (bmh-M) y Páramo: $3000 \mathrm{a}$ 4700 m.s.n.m., temperatura $10-0{ }^{\circ} \mathrm{C}$ (Suárez y Beltrán 1981).

Se establecieron 11 estaciones de muestreo, entre los 433 a 3600 m.s.n.m, cubriendo distintas zonas de vida (Cuadro 1). Basados en el ciclo hidrológico anual para el valle alto del Magdalena (Pérez 1964), las estaciones de muestreo fueron visitadas en las épocas de lluvia (marzo), transición lluvia-sequía (mayo), sequía (julio) y transición sequía-lluvia (octubre) considerando de esta forma un ciclo hidrológico completo (Cuadro 1). En los cuatro meses de estudio, las once estaciones fueron cubiertas en su totalidad en 11 días ( 1 día /estación-hábitat) para un total de 44 días de muestreo. Las mariposas se recolecciónron en tres tipos de hábitat (pradera, borde de bosque y bosque). La recolección fue al azar en transectos de longitud no definida (tipo sendero), entre las 09:00 y las 15:00 hr, empleando para ello redes lepidopterológicas de $0.5 \mathrm{~m}$ de diámetro y $1.20 \mathrm{~m}$ de profundidad, las recoleccións fueron realizadas por dos personas simultáneamente. Todos los ejemplares capturados se sacrificaron por constricción cefalotoráxica (Fagua 2001) y se guardaron en sobres de papel milano para su montaje y determinación en el Laboratorio de Investigación en Zoología de la Universidad del Tolima.

Los ejemplares fueron montados de acuerdo con el procedimiento de cámara húmeda (Fagua 2001). La determinación taxonómica se realizó siguiendo las claves de Ehrlich y Ehrlich (1961) y Andrade (1990). Adicionalmente los ejemplares se confrontaron con registros fotográficos de Anónimo (1987), De la Maza (1987) y García-Robledo et al. (2002). Con el fin de confirmar su identificación, algunos ejemplares fueron comparados con tipos Pedaliodes empusa (Felder, 1867): EN002646R, P. drymaea (Hewitson, 1858): EN002648R, Eretris calisto (C\&R. Felder, 1867): EN002649R, Lymanopoda obsoleta (Westwood, 1851): EN002650R y P. polusca (Hewitson, 1862): EN002651R), depositados en la Colección Lepidopterológica del Museo de Historia Natural de la Universidad del Cauca MHN-UC (Colombia). Todos los ejemplares recoleccióndos se encuentran en la Colección Zoológica de la Universidad del Tolima (CZUT-Ld).

Para el análisis de datos se calculó el índice de riqueza de Margalef (D) (Magurran 
CUADRO 1

Localización de las estaciones de muestreo en la cuenca del río Coello, Departamento del Tolima, Colombia

TABLE 1

Location of sampling stations in the Coello river basin, Tolima state, Colombia

\begin{tabular}{|c|c|c|c|c|c|c|}
\hline Estación & m.s.n.m & $\begin{array}{c}\text { Zona } \\
\text { de vida }\end{array}$ & $\begin{array}{l}\text { Ubicación } \\
\text { geográfica }\end{array}$ & $\begin{array}{c}\text { Fecha } \\
\text { recolección }\end{array}$ & Época climática & $\begin{array}{c}\mathrm{T}^{\circ} \text { ambiente } \\
\left({ }^{\circ} \mathrm{C}\right)\end{array}$ \\
\hline Potrerillo (Coello) & 433 & bms-T & 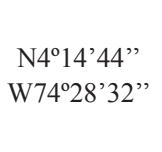 & $\begin{array}{l}03 / 20 / 03 \\
05 / 01 / 03 \\
07 / 26 / 03 \\
10 / 19 / 03\end{array}$ & $\begin{array}{c}\text { Lluvia } \\
\text { T. Lluvia-Sequía } \\
\text { Sequía } \\
\text { T. Sequía-Lluvia }\end{array}$ & $\begin{array}{l}27 \\
29 \\
31 \\
29\end{array}$ \\
\hline Buenos A (Ibagué) & 885 & bs- $\mathrm{T}$ & $\begin{array}{l}\mathrm{N} 4^{\circ} 18^{\prime} 30^{\prime \prime} \\
\text { W750' } 22^{\circ},\end{array}$ & $\begin{array}{l}03 / 28 / 03 \\
05 / 10 / 03 \\
07 / 24 / 03 \\
10 / 25 / 03\end{array}$ & $\begin{array}{c}\text { Lluvia } \\
\text { T. Lluvia-Sequía } \\
\text { Sequía } \\
\text { T. Sequía-Lluvia }\end{array}$ & $\begin{array}{l}26 \\
29 \\
32 \\
28\end{array}$ \\
\hline Martinez (Rovira) & 905 & bs- $\mathrm{T}$ & $\begin{array}{l}\mathrm{N} 4^{\circ} 17^{\prime} 22^{\prime \prime}, \\
\mathrm{W}^{\prime} 5^{\circ} 12^{\prime} 53^{\prime \prime}\end{array}$ & $\begin{array}{l}03 / 21 / 03 \\
05 / 02 / 03 \\
07 / 27 / 03 \\
10 / 20 / 03\end{array}$ & $\begin{array}{c}\text { Lluvia } \\
\text { T. Lluvia-Sequía } \\
\text { Sequía } \\
\text { T. Sequía-Lluvia }\end{array}$ & $\begin{array}{c}26 \\
28 \\
31.2 \\
29\end{array}$ \\
\hline Cerrajosa (Cajamarca) & 1641 & bh-ST & $\begin{array}{l}\text { N4²6'53"' } \\
\text { W75'22'11", }\end{array}$ & $\begin{array}{l}03 / 26 / 03 \\
05 / 07 / 03 \\
07 / 25 / 03 \\
10 / 25 / 03\end{array}$ & $\begin{array}{c}\text { Lluvia } \\
\text { T. Lluvia-Sequía } \\
\text { Sequía } \\
\text { T. Sequía-Lluvia }\end{array}$ & $\begin{array}{l}24 \\
22 \\
26 \\
23\end{array}$ \\
\hline Laureles (Ibagué) & 1661 & bh-ST & $\begin{array}{l}\mathrm{N} 4^{\circ} 22^{\prime} 24^{\prime \prime}, \\
\text { W75'22'01', }\end{array}$ & $\begin{array}{l}03 / 27 / 03 \\
05 / 09 / 03 \\
07 / 20 / 03 \\
10 / 29 / 03\end{array}$ & $\begin{array}{c}\text { Lluvia } \\
\text { T. Lluvia-Sequía } \\
\text { Sequía } \\
\text { T. Sequía-Lluvia }\end{array}$ & $\begin{array}{l}22 \\
22 \\
25 \\
23\end{array}$ \\
\hline Cay (Ibagué) & 1714 & bh-ST & $\begin{array}{l}\text { N4²9'26"' } \\
\text { W75 } 75^{\circ} 14^{\prime} 55^{\prime \prime}\end{array}$ & $\begin{array}{l}03 / 29 / 03 \\
05 / 11 / 03 \\
07 / 23 / 03 \\
10 / 28 / 03\end{array}$ & $\begin{array}{c}\text { Lluvia } \\
\text { T. Lluvia-Sequía } \\
\text { Sequía } \\
\text { T. Sequía-Lluvia }\end{array}$ & $\begin{array}{l}22 \\
23 \\
25 \\
22\end{array}$ \\
\hline La Leona (Cajamarca) & 2072 & bh-MB & 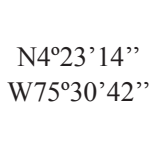 & $\begin{array}{l}03 / 23 / 03 \\
05 / 04 / 03 \\
07 / 29 / 03 \\
10 / 22 / 03\end{array}$ & $\begin{array}{c}\text { Lluvia } \\
\text { T. Lluvia-Sequía } \\
\text { Sequía } \\
\text { T. Sequía-Lluvia }\end{array}$ & $\begin{array}{l}18 \\
19 \\
20 \\
19\end{array}$ \\
\hline Toche (Cajamarca) & 2152 & bh-MB & $\begin{array}{c}\mathrm{N} 4^{\circ} 32^{\prime} 55^{\prime \prime} \\
\mathrm{W}^{\circ} 5^{\circ} 24^{\prime} 44^{\prime \prime}\end{array}$ & $\begin{array}{l}03 / 25 / 03 \\
05 / 06 / 03 \\
07 / 31 / 03 \\
10 / 24 / 03\end{array}$ & $\begin{array}{c}\text { Lluvia } \\
\text { T. Lluvia-Sequía } \\
\text { Sequía } \\
\text { T. Sequía-Lluvia }\end{array}$ & $\begin{array}{l}17 \\
16 \\
18 \\
18\end{array}$ \\
\hline Dantas (Ibagué) & 2441 & bmh-M & $\begin{array}{l}\mathrm{N} 4^{\circ} 20^{\prime} 07^{\prime \prime} \\
\text { W75'26'13"' }\end{array}$ & $\begin{array}{l}03 / 30 / 03 \\
05 / 08 / 03 \\
08 / 02 / 03 \\
10 / 26 / 03\end{array}$ & $\begin{array}{l}\text { Lluvia } \\
\text { T. Lluvia-Sequía } \\
\text { Sequía } \\
\text { T. Sequía-Lluvia }\end{array}$ & $\begin{array}{l}16 \\
15 \\
19 \\
15\end{array}$ \\
\hline Cristales (Cajamarca) & 2460 & bmh-M & $\begin{array}{l}\mathrm{N} 4^{\circ} 25^{\prime} 42^{\prime \prime} \\
\mathrm{W} 75^{\circ} 22^{\prime} 32^{\prime \prime}\end{array}$ & $\begin{array}{l}03 / 24 / 03 \\
05 / 05 / 03 \\
07 / 30 / 03 \\
10 / 23 / 03\end{array}$ & $\begin{array}{l}\text { Lluvia } \\
\text { T. Lluvia-Sequía } \\
\text { Sequía } \\
\text { T. Sequía-Lluvia }\end{array}$ & $\begin{array}{l}17 \\
15 \\
18 \\
15\end{array}$ \\
\hline Semillas (Cajamarca) & 3600 & Páramo & $\begin{array}{l}\mathrm{N} 4^{\circ} 15^{\prime} 28^{\prime \prime} \\
\text { W75'33'35", }\end{array}$ & $\begin{array}{l}03 / 22 / 03 \\
05 / 03 / 03 \\
07 / 28 / 03 \\
10 / 21 / 03\end{array}$ & $\begin{array}{c}\text { Lluvia } \\
\text { T. Lluvia-Sequía } \\
\text { Sequía } \\
\text { T. Sequía-Lluvia }\end{array}$ & $\begin{array}{c}9 \\
12 \\
13 \\
7\end{array}$ \\
\hline
\end{tabular}


1988) y el porcentaje de abundancia relativa. La diversidad total por localidad y por hábitats se analizó a través del índice de Shannon (H'), estableciéndose diferencias para las localidades y los habitats con una prueba de bondad de ajuste (t) (0.05) (Magurran 1988). De las matrices de presencia-ausencia se obtuvo índices de similitud entre estaciones según el coeficiente de Jaccard. Las matrices resultantes del procedimiento anterior fueron agrupadas mediante UPGMA (Ligamento promedio por la media aritmética no ponderada), con el programa STATISTICA (Versión 3.5, 1995), para obtener los respectivos dendrogramas de similitud.

\section{RESULTADOS}

Se recolectó un total de 239 individuos representados en cuatro tribus, 13 géneros y 34 especies. Las especies más abundantes fueron $P$. drymaea (24.26\%), P. manis (C \& R. Felder, 1867) (11.71\%), P. poesia (Hewitson, 1862) (5.43\%), Euptychoides griphe (C. \& R. Felder, 1867) (7.94\%) y Lasiophila zapatosa (Westwood, 1851) (7.53\%) (Cuadro 2).

La subfamilia Satyrinae mostró valores bajos de riqueza a menos de 1000 m.s.n.m. (D $=2.17,1.83,1.36$ ), a 1641 m.s.n.m. se presentó un pico intermedio $(\mathrm{D}=2.72)$, el cual se ve abruptamente separado a los 1661 m.s.n.m. La riqueza tiende a aumentar a los 1714 m.s.n.m, observándose un valor máximo $(\mathrm{D}=2.91)$ a 2 152 m.s.n.m. (Fig. 1).

En cuanto a la diversidad, la subfamilia Satyrinae presentó valores bajos $\left(\mathrm{H}^{\prime}=1.09 \mathrm{y}\right.$ $\left.\mathrm{H}^{\prime}=1.38\right)$ a alturas inferiores a 1000 m.s.n.m., además no existió diferencias estadísticamente significativas entre las estaciones (PotrerilloBuenos Aires: $t_{c}$ 0.82, gl 7; Potrerillo-Martínez: $\mathrm{t}_{\mathrm{c}}$ 0.38, gl 9 y Buenos Aires-Martínez: $\mathrm{t}_{\mathrm{c}}$ 0.54 , gl $3 ; \alpha 0.05$ ). Las estaciones ubicadas entre 1600 a 1800 m.s.n.m. no mostraron diferencias estadísticamente significativas (Cerrajosa-Cay: $t_{c}$ 1.53, gl 110, $\alpha$ 0.05), y registraron una diversidad intermedia $\left(\mathrm{H}^{\prime}=\right.$ 1.94) a 1641 m.s.n.m. Las estaciones entre 2000 a 2441 m.s.n.m. tampoco exhibieron
CUADRO 2

Abundancia relativa (\%) de las especies de Satyrinae, cuenca del río Coello, Departamento del Tolima, Colombia

TABLE 2

Relative abundance (\%) of the species of Satyrinae, Coello river basin, Tolima state, Colombia

\begin{tabular}{|c|c|c|c|}
\hline Tribu & Taxa & Individuos & $\% \mathrm{AR}$ \\
\hline \multirow[t]{20}{*}{ Pronophilini } & Corades chelonis & 3 & 1.255 \\
\hline & Lasiophila zapatosa & 18 & 7.531 \\
\hline & Lymanopoda albocinta & 9 & 3.765 \\
\hline & Lymanopoda obsoleta & 1 & 0.418 \\
\hline & Lymanopoda sp. Nov & 1 & 0.418 \\
\hline & Oxeoschistus simplex & 3 & 1.255 \\
\hline & Pedaliodes drymae & 58 & 24.267 \\
\hline & Pedaliodes empusa & 1 & 0.418 \\
\hline & Pedaliodes manis & 28 & 11.715 \\
\hline & Pedaliodes peucestas & 6 & 2.5104 \\
\hline & Pedaliodes phaedra & 5 & 2.092 \\
\hline & Pedaliodes poesia & 13 & 5.43 \\
\hline & Pedaliodes polusca & 2 & 0.836 \\
\hline & Pedaliodes wilhelmi & 3 & 1.255 \\
\hline & Altopedaliodes reissi & 7 & 2.928 \\
\hline & Altopedaliodes cocytia & 1 & 0.418 \\
\hline & Pronophila sp. & 1 & 0.418 \\
\hline & Steroma bega & 2 & 0.836 \\
\hline & Eretris calisto & 2 & 0.836 \\
\hline & Eretris sp. & 3 & 1.255 \\
\hline \multirow[t]{9}{*}{ Euptychiini } & Euptychia calixta & 5 & 2.092 \\
\hline & Hermeuptychia hermes & 5 & 2.092 \\
\hline & Euptychia hesione & 12 & 5.020 \\
\hline & Euptychia saturnus & 6 & 2.510 \\
\hline & Euptychia penelope & 5 & 2.092 \\
\hline & Euptychoides griphe & 19 & 7.949 \\
\hline & Oressinoma typhla & 4 & 1.673 \\
\hline & Yphthimoides sp. & 2 & 0.836 \\
\hline & Taygetis celia & 1 & 0.418 \\
\hline Erebiini & Manerebia lanassa & 1 & 0.418 \\
\hline \multirow[t]{5}{*}{ Haeterini } & Pseudohaetera hypaesia & 5 & 2.092 \\
\hline & Satyrinae sp. 17 & 3 & 1.255 \\
\hline & Satyrinae sp. 18 & 2 & 0.836 \\
\hline & Satyrinae sp. 21 & 2 & 0.836 \\
\hline & Total & 239 & 100 \\
\hline
\end{tabular}




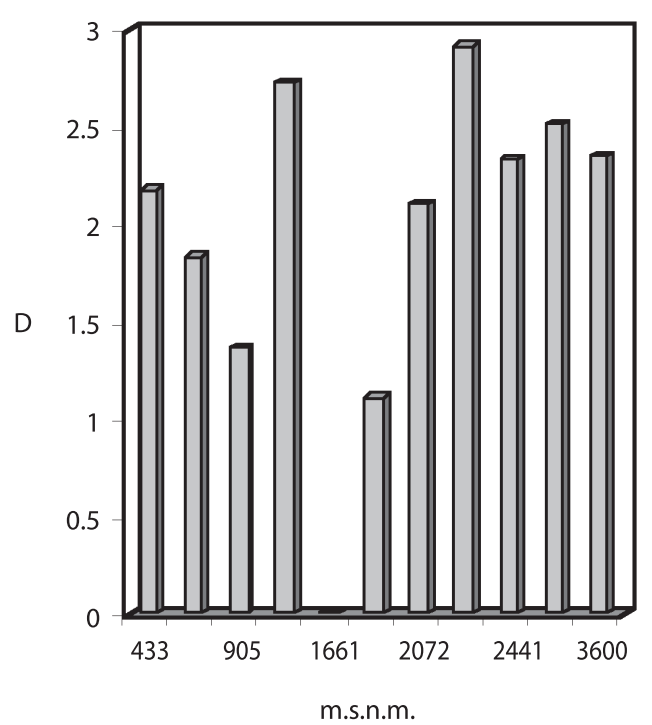

Fig. 1. Índice de Margalef (D) para la especies de Satyrinae., cuenca del río Coello, Departamento del Tolima, Colombia.

Fig. 1. Margalef index (D) for the Satyrinae species, Coello river basin, Tolima state, Colombia.

diferencias estadísticamente significativas (La Leona-Toche: $t_{c}$ 0.67, gl 48; La Leona-Dantas: $\mathrm{t}_{\mathrm{c}}$ 1.36, gl 50 y Toche-Dantas: $\mathrm{t}_{\mathrm{c}} 0.27, \mathrm{gl} 31 ; \alpha$ $0.05)$, y registraron la más alta diversidad $\left(\mathrm{H}^{\prime}=\right.$ 1.99) a una altura de 2441 m.s.n.m. Entre los 2 441 y 3600 m.s.n.m. cristales (2 460 m.s.n.m) evidencio diferencias estadísticamente significativas (Dantas-Cristales: $\mathrm{t}_{\mathrm{c}} 3.00, \mathrm{gl} \mathrm{128, \alpha}$ 0.05; Dantas-Semillas de Agua: $\mathrm{t}_{\mathrm{c}}$ 0.91, gl 36, $\alpha 0.05$ ) (Fig. 2).

La diversidad de Satyrinae en el bosque registró valores bajos $\left(\mathrm{H}^{\prime}=0.76\right.$ a $\left.\mathrm{H}^{\prime}=1.01\right)$, además no se presentó diferencias estadísticamente significativas a alturas inferiores a 1000 m.s.n.m. (Potrerillo-Buenos Aires y Potrerillo-Martínez: $\left(\mathrm{t}_{\mathrm{c}}\right.$ 0.55, gl 7, $\alpha$ 0.05). Las estaciones entre 1600 a 1800 m.s.n.m. sí presentaron diferencias estadísticamente significativas (Cerrajosa-Cay: $\mathrm{t}_{\mathrm{c}} 3.85, \mathrm{gl} 9, \alpha$ 0.05) apareciendo el valor más alto $\left(H^{\prime}=1.86\right)$ a los 1641 m.s.n.m. Por encima de 2000 hasta los 2500 m.s.n.m. se registraron valores altos de diversidad, sin presentar diferencias estadísticamente significativas entre las localidades ( $\mathrm{La}$

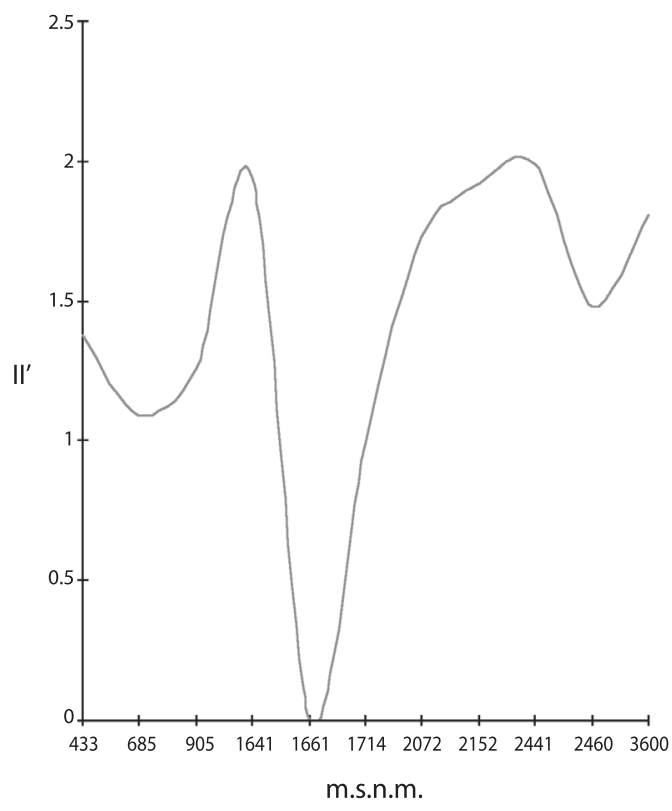

Fig. 2. Índice de Shannon ( $\left.H^{\prime}\right)$ para las especies de Satyrinae, cuenca del río Coello, Departamento del Tolima, Colombia.

Fig. 2. Shannon index (H') for the Satyrinae species, Coello river basin, Tolima state, Colombia.

Leona-Toche, La Leona-Dantas y La LeonaCristales: $t_{\mathrm{c}}$ 0.55, gl 9, $\alpha$ 0.05, Toche-Dantas y Toche-Cristales: $t_{c} 0.42, \mathrm{gl} 9, \alpha$ 0.05). En cuanto al borde de bosque, no se encontraron diferencias para las estaciones ubicadas entre $905 \mathrm{y}$ 1641 m.s.n.m, y entre 2000 y 2500 m.s.n.m, pero si se presentaron diferencias en un ámbito de 2400 a 2500 m.s.n.m (Dantas-Cristales: $\mathrm{t}_{\mathrm{c}}$ 2.36, gl 46, $\alpha$ 0.05), alcanzando el valor más alto de diversidad $\left(H^{\prime}=2.02\right)$ a los 2441 m.s.n.m. Las dos últimas estaciones no mostraron diferencias estadísticamente significativas $\left(\mathrm{t}_{\mathrm{c}} 0.33, \mathrm{gl} \mathrm{32}, \alpha 0.05\right)$. En la pradera, la diversidad se registró por encima de 2000 m.s.n.m., existiendo diferencias estadísticamente significativas a una altura de 2441 m.s.n.m. (TocheDantas: $t_{c}$ 2.07, gl 22; La Leona-Dantas: $t_{c}$ 1.84, gl 14 y La Leona-Cristales: $\mathrm{t}_{\mathrm{c}} 1.78, \mathrm{gl} 42$; $\alpha 0.05)$ donde se registró la mayor diversidad $\left(\mathrm{H}^{\prime}=1.64\right)$ (Fig. 3).

El análisis de similitud (Fig. 4) evidenció dos grupos en general. El primer grupo 


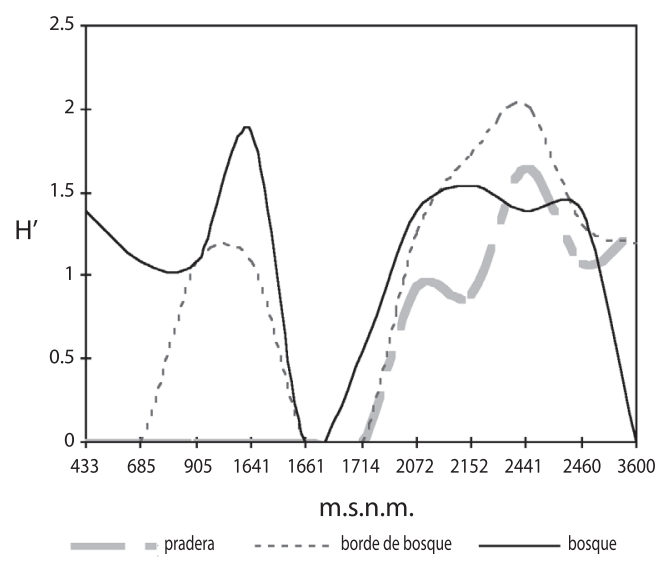

Fig. 3. Índice de Shannon Weiner (H') para Satyrinae en tres tipos de hábitat, cuenca del río Coello, Departamento del Tolima, Colombia.

Fig. 3. Shannon index (H') for Satyrinae in three types of habitat, Coello river basin, Tolima state, Colombia.

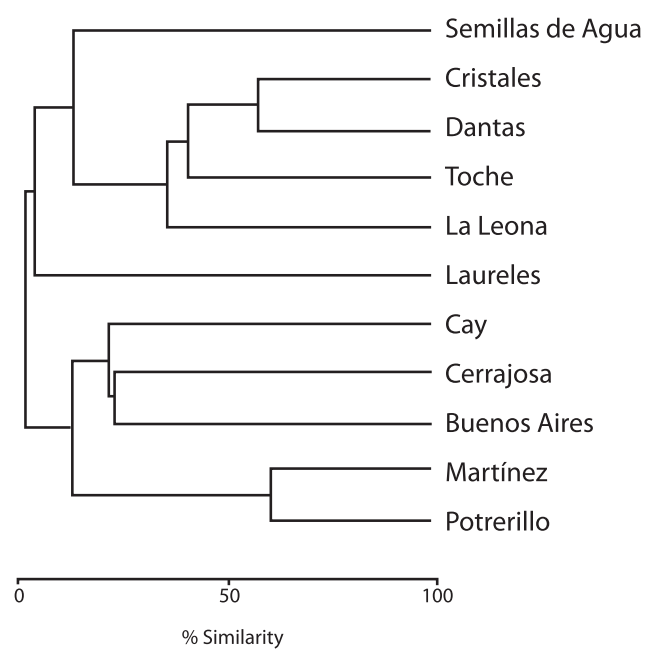

Fig. 4. Análisis de Similitud (Jaccard) para Satyrinae, cuenca del río Coello, Departamento del Tolima, Colombia. Fig. 4. Jaccard similarity analysis for Satyrinae, Coello river basin, Tolima state, Colombia.

comprendido entre 433 a 1714 m.s.n.m., está constituido por dos subgrupos. El primer subgrupo comprende las estaciones Potrerillo y Martínez, presenta la mayor similitud (60\%), mientras que el segundo mostró un porcentaje menor (Buenos Aires-Cerrajosa: 22.22\%, Buenos Aires-Cay: 20\% y Cerrajosa-Cay:
$22.22 \%$ ). El segundo grupo localizado entre 1641 a 3600 m.s.n.m., presenta un subgrupo definido entre 2172 a 2460 m.s.n.m. donde se registraron valores altos de similitud ( $\mathrm{La}$ Leona-Toche: $38.46 \%$, Toche- Dantas: $42.85 \%$ y Dantas-Cristales: 57.14\%). En este grupo las estaciones de Laureles y Semillas de Agua fueron las menos afines.

\section{DISCUSIÓN}

En la cuenca del río Coello las especies de Satyrinae representan el $35.78 \%$ de las estimadas por Andrade-C y Amat (1996) para la cordillera Central. Este número de especies es alto, ya que el área de la cuenca sólo es el 7 $\%$ del área total de la cordillera Central. El alto porcentaje de especies puede explicarse por la gran variabilidad fisiográfica y diversidad de pisos térmicos, así como las diferentes zonas de vida que generan una alta heterogeneidad en el paisaje, esté constituido a su vez, por distintos tipos de hábitat, los cuales se caracterizan por un grado específico de agregación y contraste.

En cuanto a la abundancia relativa, tres de las cinco especies más abundantes pertenecen al género Pedaliodes, el cual ha sido descrito como el más diverso de esta subfamilia (GarcíaRobledo et al. 2002). Este género es propio de tierras altas o montanas, donde se encuentran sus principales plantas hospederas (Poaceae, Cyperaceae, Selaginellaceae y Loranthaceae) (De Vries 1987).

La alta riqueza registrada a 2152 m.s.n.m $(\mathrm{D}=2.91)$ puede explicarse bajo la hipótesis de "domino intermedio" (Colwell y Less 2000). Es decir, que este valor es producto de la convergencia o solapamiento de rangos de distribución altitudinal de las especies hacia el centro del dominio (altura intermedia), debido a la extensión de dichos rangos de las especies confinadas tanto en zonas montanas como bajas.

El patrón de diversidad también evidenció un alto valor en zonas montanas, a los 2441 m.s.n.m $\left(\mathrm{H}^{\prime}=1.99\right)$, esto debido, a respuestas biológicas que permiten tolerar condiciones extremas de frío, humedad y radiación solar. 
Además de la neblina, la cual tiende a aumentar la humedad ambiental y a decrecer la evapotranspiración (Anónimo 2004), factor que se encuentra relacionado con la productividad primara (Rosenzweig 1968). García-Robledo et al. (2002) mencionan que las especies de Satyrinae, y en especial de la tribu Pronophilini, son más diversas entre 2600 y 2700 m.s.n.m. donde se encuentra una gran cantidad de especies endémicas para las tres cordilleras colombianas, y Pyrcz y Wojtusiak (2001) informan una gran diversidad de ésta tribu a 2700 m.s.n.m. en la cordillera de Mérida (Venezuela). Por otra parte, muchas especies de Satyrinae tienen como plantas hospederas miembros de las familias Poaceae y Selaginallaceae, así como Briófitos (De Vries 1987), estos últimos son predominantes entre 2000 y 2400 m.s.n.m. (selva andina) (Anónimo 2004). Es posible que algunas especies del género Pedaliodes prefieran el chusque (Chusquea scadens) como la planta nutricia de sus larvas (Anónimo 1987), siendo ésta gramínea indicadora de formación vegetal montana (Esquivel 1993).

Los resultados demuestran que ocurre un aumento de la riqueza y diversidad de Satyrinae a medida que se asciende altitudinalmente, lo cual contradice la "regla de Rapoport" (Stevens 1992, Blackburn y Gaston 1996). Éste comportamiento podría deberse a que el $69.87 \%$ de las especies registradas en la cuenca del río Coello pertenecen a la tribu Pronophilini, lo cual estaría de acuerdo con lo propuesto por Pyrcz (2004) quien menciona que aproximadamente el 95\% de las especies de Pronophilini se encuentran en los Andes, siendo este grupo de Satyrinae altamente diversificado en zonas montanas y de páramo.

El hábitat con mayor diversidad fue el borde de bosque $\left(\mathrm{H}^{\prime}=2.02\right)$, seguido por el bosque $\left(\mathrm{H}^{\prime}=1.86\right)$ y la pradera $\left(\mathrm{H}^{\prime}=1.64\right)$. La incidencia de una alta diversidad de Satyrinae, tanto en praderas como en bordes de bosque, puede reflejar que éstos hábitats son óptimos para especies como Euptychia hesione (Sulzer, 1776) y Hermeuptychia hermes (Fabricius, 1775) las cuales acostumbran plantas de las familias Asteraceae y Poaceae (Álvarez 1993), frecuentes en zonas de transición y de pradera, en comparación a las especies de bosque las cuales están asociadas a estructuras de vegetación más desarrollada y de composición florística diversa. Tobar (2000) propone una tendencia de especies de mariposas generalistas en hábitats de pradera con algún tipo de perturbación o transformación, mientras que las especies de bosque, tienden a ser especialistas y prefieren hábitats con estructuras de vegetación más complejas. En este estudio, el bosque exhibía relictos de parches con diferentes estados de sucesión primaria y secundaria, lo cual probablemente deriva en una alta heterogeneidad en los estratos y posibilita un mayor número de microhábitats para algunas especies, como Pseudohaetera hypaesia (Hewitson, 1854) propia del interior de bosque (Álvarez 1993) y que muestra afinidad por el estrato subarbustivo y Taygetis celia, (Cramer, 1779) indicadora de bosque secundario (Álvarez 1993) donde vuela a la altura del estrato herbáceo y rasante, el cual aprovecha debido a su coloración críptica para pasar desapercibidas junto a la hojarasca del suelo.

En el análisis de similitud para el primer grupo, el subgrupo Potrerillo-Martínez mostró el valor más alto $(60 \%)$, debido posiblemente al mayor número de especies comunes y abundantes como $E$. hesione y $H$. hermes, frecuentes en hábitats abiertos y zonas de transición (Álvarez 1993). La baja similitud del subgrupo Buenos Aires-Cerrajosa-Cay es posiblemente debida a la presencia de especies raras como $P$. hypaesia y $T$. celia, propias del interior de bosque (Álvarez 1993). En el segundo grupo, el subgrupo La Leona-Toche-Dantas-Cristales mostró altos valores de similitud, posiblemente por la incidencia de especies comunes y abundantes en zonas frías y de Páramo como P. drymaea, P. manis, P. poesia, E. griphe y L. zapatosa, además de especies raras como L. obsoleta, P. polusca y E. calisto. Las localidades de Laureles y Semillas de Agua, no obedecieron a ningún agrupamiento debido al muy bajo número de especies comunes.

Este estudio representa un primer paso para el conocimiento de la diversidad y composición 
anual de las mariposas Satyrinae en la cuenca del río Coello, registrándose las especies $P$. hypaesia y $T$. celia como propias del interior de bosque y L. obsoleta, P. polusca y E. calisto como indicadoras de zonas montanas y de páramo. Es importante futuros estudios de monitoreo de éstas especies y sus relaciones tróficas dentro de los ecosistemas andinos.

\section{AGRADECIMIENTOS}

Los autores agradecen a Santiago Ayerbe, por la colaboración prestada en el Museo de Historia Natural de la Universidad del Cauca (MHN-C) (Colombia), e igualmente a Giovany Guevara Cardona, por la revisión del texto.

\section{RESUMEN}

Este estudio describe los patrones de diversidad y distribución de la subfamilia Satyrinae en la cuenca del

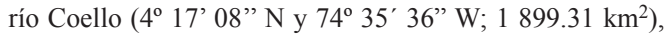
en un gradiente altitudinal de 433 a 3600 m.s.n.m, con el fin de determinar la diversidad y composición regional y local de este grupo de mariposas y su posible uso como indicador biológico de zonas montanas y de Páramo. Se establecieron 11 estaciones de muestreo las cuales incluyen distintas zonas de vida. Las muestras se recolecciónron durante un periodo de 11 días en marzo, mayo, julio y octubre de 2003, en tres tipos de hábitat (bosque, borde de bosque y pradera). La recolección fue al azar en transectos de longitud no definida, entre las 09:00 y las 15:00 hr, empleando para ello redes lepidopterológicas de $0.5 \mathrm{~m}$ de diámetro y $1.20 \mathrm{~m}$ de longitud. Para el análisis de datos se calculó el índice de riqueza de Margalef (D) y se registró el porcentaje de abundancia relativa para las especies. La diversidad total por localidad y por hábitat se analizó a través del índice de Shannon (H'). De las matrices de presencia-ausencia se obtuvo índices de similitud entre estaciones, según coeficiente de Jaccard. Se recolectó un total de 239 individuos de 13 géneros y 34 especies. Las especies más abundantes fueron especialmente del género Pedaliodes, representando el $41.4 \%$. La riqueza y diversidad, presentaron un comportamiento similar, mostrando altos valores en zonas montanas y de Páramo $(>2000$ m.s.n.m). En cuanto a la diversidad por hábitat, se observó la mayor diversidad en el borde de bosque. El análisis de similitud evidenció dos grupos claramente definidos. El primer grupo contempla especies propias de zonas cálidas (Euptychia hesione y Hermeuptychia hermes) y templadas (Pseudohaetera hypaesia y Taygetis celia), mientras el segundo presenta especies exclusivas de zonas montanas y de Páramo (Lymanopoda obsoleta, Pedaliodes polusca y Eretris calisto).

Palabras clave: Coello, Satyrinae, Pedaliodes, zonas montanas, páramo, similitud.

\section{REFERENCIAS}

Álvarez, J. 1993. Inventario de las mariposas (Lepidoptera: Rhopalocera), Con anotaciones ecológicas, para dos zonas del Departamento de Risaralda, Colombia. Tesis de pregrado, Universidad Nacional, Bogotá, Colombia. 204 p.

Amat, G., F. Fernández \& G. Andrade-C. 1999. Un vistazo actual a la taxonomía de insectos en Colombia (Coleoptera, Himenóptera y Lepidoptera). Insectos de Colombia. 1: 14-33.

Andrade, G. 1990. Clave para las familias y subfamilias de Lepidoptera: Rhopalocera de Colombia. Caldasia. 16: 539-550.

Andrade, G. \& G. Amat. 1996. Estudio regional de las mariposas altoandinas en la cordillera Oriental de Colombia. Insectos de Colombia. 1: 149-180.

Anónimo. 1987. El encanto de las mariposas de Colombia. Renaser, Bogotá, Colombia. 64 p.

Blackburn, T. M. \& K. J. Gaston. 1996. Spatial patterns in the geographic range sizes of bird species in the new world. Philos. Trans. R. Soc. Lond. B 351: 897-912.

Colwell, R. K. \& D. C. Less. 2000. The middomain effect: Geometric constraints on the geography of species richness. Trends Ecol. Evol. 15: 70-76.

De la Maza, R. 1987. Mariposas Mexicanas. Fondo de cultura Económica, México DF. 301 p.

De Vries, P. J. 1987. The butterflies of Costa Rica and their Natural History. Papilionidae. Pieridae. Nymphalidae. Princeton, Nueva Jersey, EEUU. 327 p.

Erhlich P. R. \& A. H. Ehrlich. 1961. How To Know The Butterflies. W.M. C. Brown, Iowa, EEUU. 262 p.

Esquivel, H. E. 1993. Taxonomía de las angiospermas (Magnoliópsidas y liliopsídas. Universidad del Tolima, Colombia. 485 p.

Fagua, G. 1999. Variación de las mariposas y hormigas de un gradiente altitudinal de la cordillera Oriental (Colombia). Insectos de Colombia. 2: 318-363. 
Fagua, G. 2001. Manual de metodologías para el desarrollo de Inventarios y Monitoreo de la Biodiversidad: Mariposas diurnas (Lepidoptera). Grupo de Exploración y Monitoreo ambiental, Bogotá, Colombia. 536 p.

García-Robledo, L., M. Constantino, M. Dolores \& G. Kattan. 2002. Mariposas comunes de la cordillera Central de Colombia. Feriva, Colombia. 130 p.

Kremen, C., R. K. Colwell, T. L. Erwin, D. D. Murphy, R. F. Noss \& M. A. Sanjayan. 1993. Terrestrial arthropod assemblaeges: their use in conservation planning. Cons. Biol. 7: 796-808.

Magurran, A. E. 1988. Diversidad ecológica y su medición. Vedra. Córcega. Princeton, Nueva Jersey, EEUU.

Pérez, A. 1964. El ciclo anual de las lluvias en Colombia (según E. Guhl).p. 247. In A. Codazzi (ed.). Recursos Naturales de Colombia, su génesis, su medida, su aprovechamiento, conservación y renovación. Dificultades naturales de Colombia y lucha contra ellas. Bogotá, Colombia.

Pyrcz, T. W. 2004. Pronophiline butterflies of the highlands of Chachapoyas in northern Peru: faunal survey, diversity and distribution patterns (Lepidoptera, Nymphalidae, Satyrinae). Genus 15: 455-622.
Pyrcz, T. W. \& J. Wojtusiak. 2001. The vertical distribution of pronophiline butterflies (Nymphalidae: Satyrinae) along and elevational transect in Monte Zerpa (Cordillera de Mérida, Venezuela) with remarks on their diversity and parapatric distribution. Global Ecol. Biogeog. 11: 211.

Rosenzweig, P. J. 1968. Net primary productivity of terrestrial environments: predictions from climatological dad. Am. Nat. 102: 67-84.

Stevens, G. C. 1992. The elevational gradient in altitudinal range: and extrension of Rapoport's latitudinal rule to altitude. Am. Nat. 140: 893-911.

Suárez, R. \& Beltrán, G. 1981. Plan de Protección y Saneamiento Ambiental en la cuenca del río Coello. Cortolima, Colombia. 327p.

Tobar, L. 2000. Diversidad de mariposas (Lepidoptera: Rhopalocera) en la parte alta del río Roble (Quindío - Colombia). Tesis de grado, Pontificia Universidad Javeriana, Bogotá. 182 p.

\section{REFERENCIA DE INTERNE T}

Anónimo. 2004. Bosque seco/bosque subandino/bosque (http://www.sentir.org/zonas/index.html; consultada 15 marzo 2004). 
